\title{
PLANARITY IN ALGEBRAIC SYSTEMS
}

BY MICHAEL ANSHEL AND JAMES R. CLAY ${ }^{1}$

Communicated by R. S. Pierce, March 8, 1968

Planarity was introduced into algebra by Marshall Hall in his wellknown coordinatization of a projective plane by a planar ternary ring [4]. In [6], J. L. Zemmer defines a near-field to be planar when the equation $a x=b x+c$ has a unique solution for $a \neq b$. In our investigation of planarity, we discovered that if $(N,+, \cdot)$ is a near-ring satisfying the above equational property, then $(N,+, \cdot)$ is a near-field. (This was conjectured by both D. R. Hughes and J. L. Zemmer in private communications.) We present some extensions of this result together with geometric interpretations of "planar" near-rings.

Definitions and notations. By a left distributive system is meant a triple $(N,+, \cdot)$ such that multiplication - is left distributive over addition + . Elements $a, b \in N$ are called left equivalent multipliers, denoted by $a \equiv_{m} b$ iff $a x=b x$ for all $x \in N$. The relation $\equiv_{m}$ is discrete when $a \equiv_{m} b$ implies $a=b$. A left distributive system is said to possess the planar property if the equation $a x=b x+c$ has a unique solution for $a \neq \neq_{m} b$.

Definition. A left distributive system $(N,+, \cdot)$ with planar property is a planar system if

(1) in $(N,+)$ the right cancellation law is valid;

(2) in $(N,+)$ there is an identity 0 ;

(3) $(N, \cdot)$ is a semi-group;

(4) there are at least three points in $N$, no two of which are left equivalent multipliers.

A planar system is integral if 0 is the only left zero divisor.

Main results. Let $(N,+, \cdot)$ be an integral planar system. Then $0 \cdot x=x \cdot 0=0$ for all $x \in N$. Let $1_{a}$ be the solution to the equation $a \cdot x=a, a \neq 0$, and $B_{a}=\left\{x \in N^{*} \mid x \cdot 1_{a}=x\right\}$, where $N^{*}$ denotes the nonzero elements of $N$. We have the following

Theorem 1. Let $(N,+, \cdot)$ be an integral planar system. Then

(i) each $\left(B_{a}, \cdot\right)$ is a group with identity $1_{a}$;

(ii) the family $\left\{B_{a}\right\}_{a \in N^{*}}$ is pairwise disjoint;

(iii) $N^{*}=\bigcup_{a \in N^{*}} B_{a}$;

1 The second author received support from NSF contract \#GP-2141. 
(iv) $N^{*} B_{a}=B_{a}$ for each $a \in N^{*}$;

(v) if $a, c \in N^{*}$, then $\phi: B_{a} \rightarrow B_{c}$ defined by $\phi(x)=x 1_{c}$ is an isomorphism;

(vi) each $1_{a}$ is a left identity for $(N,+, \cdot)$.

CoRollary. Let $(N,+, \cdot)$ be a near-ring that is an integral planar system with $\equiv_{m}$ discrete. Then $(N,+, \cdot)$ is a planar near-field.

Proof. If $a, b \in N^{*}$, then $1_{a} \equiv_{m} 1_{b}$.

In the sequel a near-ring that is an integral planar system will be called an integral planar near-ring.

THEOREM 2. Suppose $(N,+, \cdot)$ is an integral planar near-ring and each $\bar{B}_{a}=\{0\} \cup B_{a}$ is an additive normal subgroup. Also suppose that no $\bar{B}_{a}=N$ but any two $\bar{B}_{a}, \bar{B}_{c}$ generate $N$ under + . Then

(i) each $\left(\bar{B}_{a},+, \cdot\right)$ is a near-field;

(ii) $\left(\bar{B}_{a},+, \cdot\right)$ is isomorphic to $\left(\bar{B}_{c},+, \cdot\right)$ if $(x+y) 1_{c}=x 1_{c}+y 1_{c}$ for all $x, y \in B_{a}$;

(iii) $(N,+)$ is abelian and is isomorphic to the direct sum $\bar{B}_{a} \oplus \bar{B}_{c}$ as groups;

(iv) the points of $N$ are the points of an affine plane $A$ with the cosets of the $\bar{B}_{a}$ as lines;

(v) the plane A can be coordinatized by a skew field.

Proof. The group $(N,+)$ is a $\Phi(\mathrm{I}, \mathrm{IV})$ group [5]. A $\Phi(\mathrm{I}, \mathrm{IV})$ group is abelian since $x \rightarrow x+g$ induces a translation on $A$ and so Axiom 4a is satisfied (p. 58 of [1]). Axiom $4 \mathrm{bP}$ (p. 63 of [1]) holds at $0 \in N$ where $x \rightarrow t x$ are the required dilatations.

Theorem 3. Suppose $(N,+, \cdot)$ is a finite integral planar near-ring and each $\bar{B}_{a}=\{0\} \cup B_{a}$ is an additive subgroup. Also suppose that no $\bar{B}_{a}=N$ but any two $\bar{B}_{a}, \bar{B}_{c}$ generate $N$ under + . Then

(i) $(N,+)$ is abelian;

(ii) the affine plane $A$ determined by $N$ can be coordinatized by a field $(F,+, \cdot)$;

(iii) each $\left(\bar{B}_{a},+, \cdot\right)$ is a field;

(iv) each $B_{a}=\{(x, m x) \mid x \in F\}$ for some $m \in F$, or $B_{a}$ $=\{(0, x) \mid x \in F\}$.

Proof. Each $\left(\bar{B}_{a},+, \cdot\right)$ is a near-field, hence $(N,+)$ is a $p$-group. Now $\left(\bar{B}_{a},+\right)$ is contained in the center of $(N,+)$ for some $a \in N^{*}$, hence $(N,+)$ is abelian since $N=\bar{B}_{a}+\bar{B}_{c}$. A finite skew field is a field, and each $\left(\bar{B}_{a},+, \cdot\right)$ is isomorphic to the coordinization skew field.

Examples. 1. Let $(F,+, \cdot)$ be a field. Define $+_{\lambda}(\lambda \neq 0)$ by $a+{ }_{\lambda} b=b$ if $a=0, a+{ }_{\lambda} b=a+(\lambda b)$ when $a \neq 0$. Then $\left(F, t_{\lambda}, \cdot\right)$ is a nontrivial 
integral planar system where $\equiv_{m}$ is discrete and $t_{\lambda}$ is not necessarily associative.

2. Let $(R \times R,+)$ be additive group of complex numbers. Define by $(a, b) \cdot(c, d)=\|(a, b)\|(c, d)$ where $\|\cdot\|$ is any norm on $R \times R$. Then $(R \times R,+, \cdot)$ is an integral planar near-ring.

3. Let $(R \times R,+)$ be as in 2 . Define $\cdot$ by $(a, b) \cdot(c, d)=(a, b)^{\wedge}(c, d)$ where $(a, b)^{\wedge}=0$ if $a=b=0$; otherwise $(a, b)^{\wedge}$ is the first nonzero coordinate. Then $(R \times R,+, \cdot)$ is an integral planar near-ring.

4. Let $(R \times R,+)$ be as in 2. Define $*$ by $(a, b) *(c, d)=$ $(a, b) / \mid) a, b) \mid \cdot(c, d)$ where $|(a, b)|=\left(a^{2}+b^{2}\right)^{1 / 2} \neq 0$ and $\cdot$ denotes the usual multiplication of complex numbers. If $(a, b)=(0,0)$, then $(a, b) *(c, d)=(0,0)$. Then $(R \times R,+, \cdot)$ is an integral planar near-ring.

5. Table 1 defines a multiplication $\cdot$ on the cyclic group $\left(Z_{5},+\right)$ such that $\left(Z_{5},+, \cdot\right)$ is an integral planar near-ring. Note that $B_{1}$ $=\{1,4\}, B_{2}=\{2,3\}$. Define $\bar{B}_{i}=B_{i} \cup\{0\}$ and $B_{i j}=\bar{B}_{i}+j, i=1,2$; $j \in Z_{5}$. If

\begin{tabular}{l|lllll}
$\cdot$ & 0 & 1 & 2 & 3 & 4 \\
\hline 0 & 0 & 0 & 0 & 0 & 0 \\
1 & 0 & 1 & 2 & 3 & 4 \\
2 & 0 & 4 & 3 & 2 & 1 \\
3 & 0 & 1 & 2 & 3 & 4 \\
4 & 0 & 4 & 3 & 2 & 1
\end{tabular}

TABLE 1

we let $I=Z_{5}$, then the $B_{i j}$ are circles of an inverse plane [3]. This example was obtained using a digital computer. (See [2].)

It is of interest to graph the left identities and the $B_{a}$ in each of the Examples 2, 3, and 4.

\section{BIBLIOGRAPHY}

1. E. Artin, Geometric algebra, Interscience, New York, 1957.

2. J. R. Clay, The near-rings on groups of low order, Math. Z. 104 (1968), 364-371.

3. P. Dembowski and D. R. Hughes, On inversive planes, J. London Math. Soc. 40 (1965), 171-182.

4. M. Hall, Jr., Projective planes, Trans. Amer. Math. Soc. 54 (1943), 229-277.

5. V. P. Zarovnyi, Interpretation of the plane axioms of affine geometry in an abstract group, Ukrain. Mat. Ž. 10 (1958), 351-364.

6. J. L. Zemmer, Near-fields, planar and nonplanar, The Math Student 31 (1964), 145-150.

UNIVERSITY OF ARIZONA 